\title{
Risques, dérives, faisabilités du travail d'obstacles en didactique
}

\section{Brigitte Peterfalvi et Anne Vérin}

\section{(2) OpenEdition \\ 1 Journals}

\section{Édition électronique}

URL : http://journals.openedition.org/trema/2066

DOI : 10.4000/trema.2066

ISSN : 2107-0997

\section{Éditeur}

Faculté d'Éducation de l'université de Montpellier

\section{Édition imprimée}

Date de publication : 1 septembre 1996

Pagination : 123-136

ISSN : 1167-315X

\section{Référence électronique}

Brigitte Peterfalvi et Anne Vérin, «Risques, dérives, faisabilités du travail d'obstacles en didactique », Tréma [En ligne], 9-10 | 1996, mis en ligne le 18 septembre 2013, consulté le 19 avril 2019. URL : http:// journals.openedition.org/trema/2066 ; DOI : 10.4000/trema.2066

Ce document a été généré automatiquement le 19 avril 2019.

Trema 


\title{
Risques, dérives, faisabilités du travail d'obstacles en didactique
}

\author{
Brigitte Peterfalvi et Anne Vérin
}

\section{Introduction}

1 À côté des présentations de résultats de recherche, il peut y avoir place, dans une rencontre comme celle-ci, pour des présentations de problématiques ou de choix épistémologiques fortement dépendants de l'objet choisi pour la recherche, des conséquences et des interrogations qui en découlent. Dans notre esprit, ce type de présentation prépare le terrain pour la constitution de groupes de travail que l'Association Européenne de Didactique de la Biologie est bien placée pour impulser, et qui permettront des collaborations de recherche entre différents pays sur des thèmes communs.

2 Pour réfléchir ensemble à certaines spécificités de la recherche didactique, nous exposerons non pas des résultats, mais le fonctionnement d'une recherche, en questionnant les limites des ambitions que peut avoir cette recherche, les types d'analyses possibles, le statut des résultats produits et leur utilité pratique et théorique.

\section{Objet de recherche et choix épistémologiques}

3 Cette recherche se proposait d'éprouver la fécondité pratique du concept d'« objectifobstacle ", proposé par Martinand en 1986. Ce concept, élaboré d'abord sur un plan théorique, à partir de théories de l'apprentissage, de réflexions épistémologiques et de considérations sur les tentatives de la pédagogie par objectifs, a permis de renouveler l'intérêt pour les représentations des élèves, en esquissant une stratégie pour leur prise en compte dans les activités didactiques. Il a proposé des pistes pour résoudre conjointement ce problème avec celui de l'émiettement des objectifs rencontré dans les perspectives de la Pédagogie par Objectifs. Se donner comme objectif le franchissement 
de quelques obstacles sélectionnés, correspondant à des progrès décisifs dans les apprentissages conceptuels, devait fournir aux enseignants des repères pour mieux comprendre le mode de fonctionnement des représentations de leurs élèves et orienter leur action éducative. Malgré sa préoccupation pragmatique de fournir des outils utilisables, cette proposition n'avait donné lieu jusque là qu'à peu de réalisations pratiques. La mise en œuvre effective de situations d'enseignement s'appuyant sur ce cadre théorique devait contribuer à en préciser les contours et à explorer les modalités possibles de la prise en compte des obstacles dans l'enseignement. Elle devait contribuer à en cerner les implications, qui restent nécessairement inaperçues tant que la façon dont la réalité résiste aux propositions théoriques n'a pas été éprouvée.

4 Ce projet impliquait un mode d'association particulier avec des enseignants. Une vingtaine d'enseignants ont été invités à construire et expérimenter des séquences d'enseignement dans leurs classes, à partir d'un cadre théorique de départ et d'une réflexion commune sur la nature des obstacles et les stratégies possibles pour leur dépassement par les élèves. Leur part importante clans l'élaboration des situations de travail et leur association aussi étroite que possible à la constitution d'un cadre théorique commun devait assurer à la fois l'insertion des séquences expérimentées dans le projet et leur compatibilité avec les conditions qu'impose le système scolaire. C'était en effet nécessaire pour éprouver la faisabilité scolaire du projet.

Le choix épistémologique de cette recherche est représenté dans le schéma de la figure 1 par la colonne de droite. Nous n'avons pas retenu une option expérimentaliste, représentée par la colonne de gauche, dont le but serait principalement de tester la faisabilité intellectuelledu travail sur les obstacles par les élèves et l'efficacité de tel ou tel procédé dans ce but. Elle aurait conduit à établir à l'avance des protocoles de travail que les enseignants auraient été invités à « exécuter ». La cohérence des situations proposées avec le cadre théorique de départ aurait été de ce fait davantage garantie, puisqu'on se situait dans une perspective d' " application » du cadre théorique. Cependant de nouveaux problèmes se seraient posés. Par exemple l'adhésion des enseignants au projet aurait été moindre, or celle-ci est particulièrement importante dans la perspective d'enseignement proposée. Nous adoptons une perspective qui vise plutôt à cerner la faisabilité scolaire del'idée d'objectif-obstacle. De ce fait, notre paradigme de recherche prend en compte une plus grande complexité. Outre les possibilités intellectuelles pour les élèves du dépassement des obstacles à l'apprentissage de tel ou tel concept, il prend en compte les résistances du système scolaire à ce type de travail. La perspective choisie conduit à entretenir des relations beaucoup plus dialectiques entre cadre théorique propositionnel et réalisation concrète. 


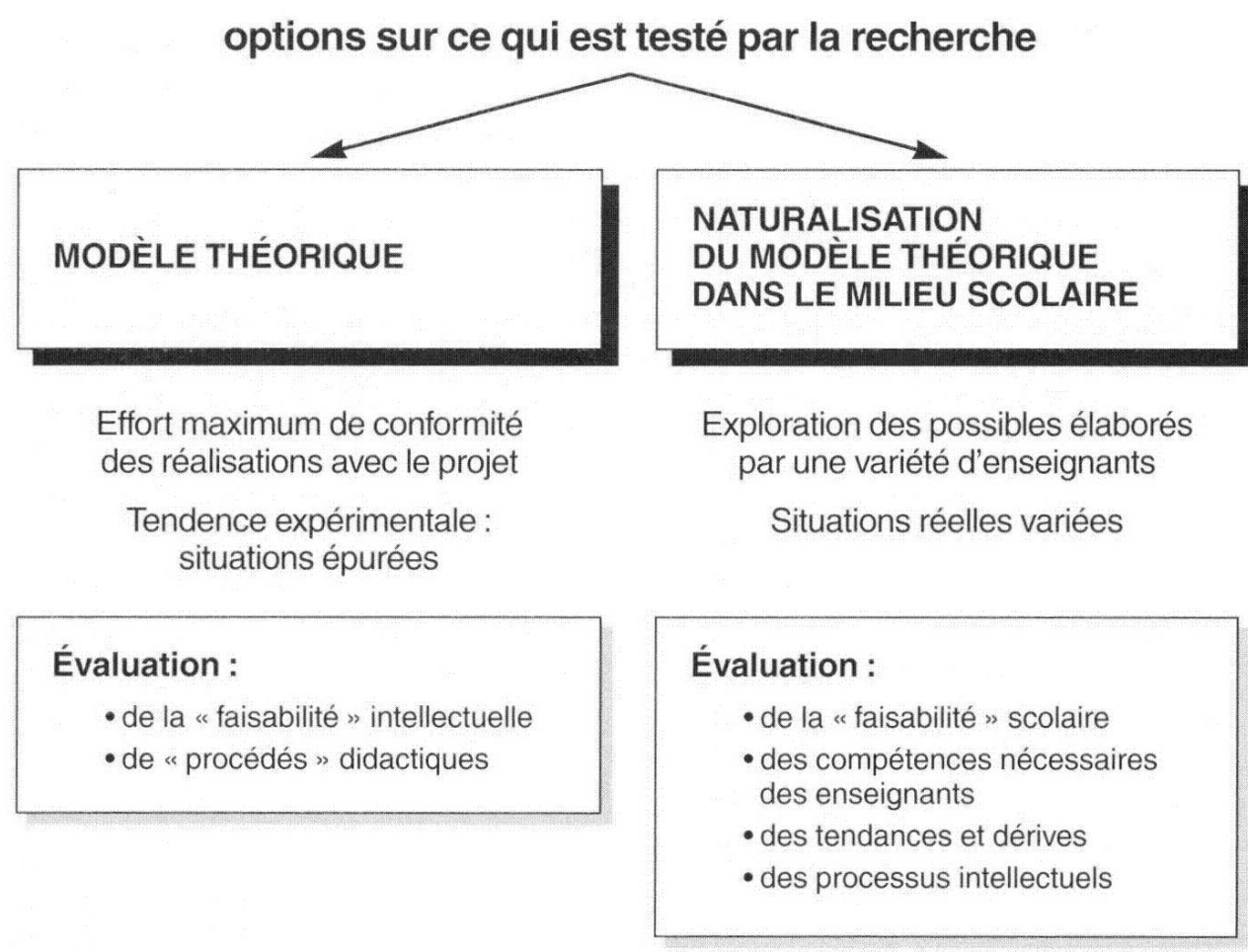

La mise en œuvre d'un tel dispositif de travail a abouti à la construction d'une grande diversité de situations, qui s'intégraient de façon plus ou moins cohérente dans le cadre conceptuel de départ. L'analyse de scénarios établis par les enseignante avant la mise en œuvre des situations, du déroulement effectif des séquences, du contenu des discussions du groupe de recherche, et d'entretiens avec les enseignants, nous ont permis de repérer un certain nombre de décalages ou de dérives par rapport au projet théorique et de réfléchir aux limites de la faisabilité de ce projet dans la réalité de l'enseignement.

En effet, même si elle implique une modification importante des modalités habituelles d'enseignement, la perspective de travail par «objectifs-obstacles » reste soumise aux mêmes contraintes. Les séquences produites sont donc la résultante d'une élaboration théorique et d'un faisceau de contraintes de plusieurs ordres qui en déterminent le caractère réalisable : contraintes institutionnelles, représentations de la discipline et des processus d'enseignement/apprentissage chez les enseignants, problèmes liés à la gestion de la classe, attitudes des élèves. Les interprétations de l'évolution des idées des élèves doivent prendre en compte cette complexité et sont différentes de celles qu'on aurait pu tirer de situations plus épurées.

\section{Décalages : compromis nécessaires ou dérives?}

8 Le projet théorique précisait certaines caractéristiques de la dynamique de fonctionnement des obstacles dont il était pertinent de tenir compte dans la conception des situations didactiques: leur résistance au changement, liée à la fonction positive qu'ils jouent en tant que système d'idées conférant une intelligibilité aux phénomènes, leur transversalité par rapport à plusieurs contenus, leur structure en réseaux, leur caractère multiforme. Il optait pour quelques principes fondamentaux dans leur 
traitement didactique: jeu sur une complémentarité entre déconstruction des idées en place et (re)construction des concepts visés; prise en compte des aspects sociaux et affectifs mis en jeu dans ce renversement des cadres de pensée, délimitation de moments plus spécifiquement centrés sur le travail des obstacles. En cela, il s'agissait d'un cadre à la fois théorique et propositionnel.

Ce cadre général laissait toutefois une latitude importante quant aux modalités précises du travail didactique, ce qui explique la grande diversité des situations explorées. Cependant, dans certains cas, les situations proposées débordent ce cadre général. Ces décalages, qui révèlent la résistance du système scolaire au projet, se manifestent :

- dans le mode de construction des séquences par les enseignants, par exemple par le rôle privilégié donné aux contradictions logiques, alors que beaucoup de choses se jouent sur un plan moins rationnel en ce qui concerne les obstacles ;

- dans la gestion de ces séquences, lorsque l'enseignant autorise plus ou moins effectivement l'expression de l'erreur et du doute - nous en verrons un exemple plus loin - ou encore lorsque son respect scrupuleux d'un protocole préétabli l'éloigne de l'écoute des élèves

- dans les réactions d'étonnement ou d'inquiétude par rapport aux processus intellectuels qui apparaissent dans ces séquences (évitements des conflits, régressions apparentes...) ;

- et même parfois, mais ceci est une situation limite, par des séquences où on ne repère plus vraiment de travail sur les obstacles.

Ces décalages, que l'on peut qualifier de dérives lorsqu'ils ne sont plus en cohérence avec le projet, deviennent à leur tour objet d'analyse dans la recherche. Il convient alors de s'interroger sur leur caractère évitable. Dans les cas où on peut penser qu'il s'agit de décalages incontournables, de compromis nécessaires, c'est le cadre propositionnel qu'il est pertinent de remanier ou de moduler. Dans d'autres cas, les analyses conduisent à l'élaboration de ripostes dans les principes de construction des séquences ou dans les modalités d'intervention des enseignants. La plupart du temps, ces décalages révèlent des points de tension pour l'enseignant, qui doit prendre en compte dans une même action le projet de travail sur les obstacles et les exigences du système scolaire, vécus comme contradictoires. On peut les rapporter à des contraintes institutionnelles (par exemple, la nécessité de donner des notes, plus ou moins fréquemment selon les établissements), à des nécessités de gestion de la classe (sauf à l'école primaire où la souplesse est plus grande, le cadre de l'heure ou des deux heures de cours doit former une unité achevée, qui supportera une semaine d'interruption). Il nous semble que les conceptions de l'apprentissage et de l'enseignement généralement partagées dans le contexte social et les conceptions privées des enseignants impliqués dans cette recherche peuvent également jouer un rôle explicatif.

11 L'exemple qui suit peut en donner une idée. Après une phase où la majorité des élèves d'une classe de sixième exprime l'idée que le dioxyde de carbone est un gaz respiratoire nocif pour l'organisme et que comme tel il ne peut pas constituer une source de matière pour la croissance de tomates, un document est distribué qui relate une expérience de l'INRA et montre que, contrairement à la prévision des élèves, l'augmentation du taux de dioxyde de carbone dans l'air des serres conduit à une augmentation de la masse des tomates cultivées. L'un des élèves, Pierre-Yves, ne peut pas accepter cette idée. Lorsque l'enseignante lui demande de lire le document à haute voix, il s'en montre incapable. « C'est impossible, dit-il, puisque le $\mathrm{CO}_{2}$ est le gaz rejeté. » C'est finalement un autre élève qui lit le document à sa place. 


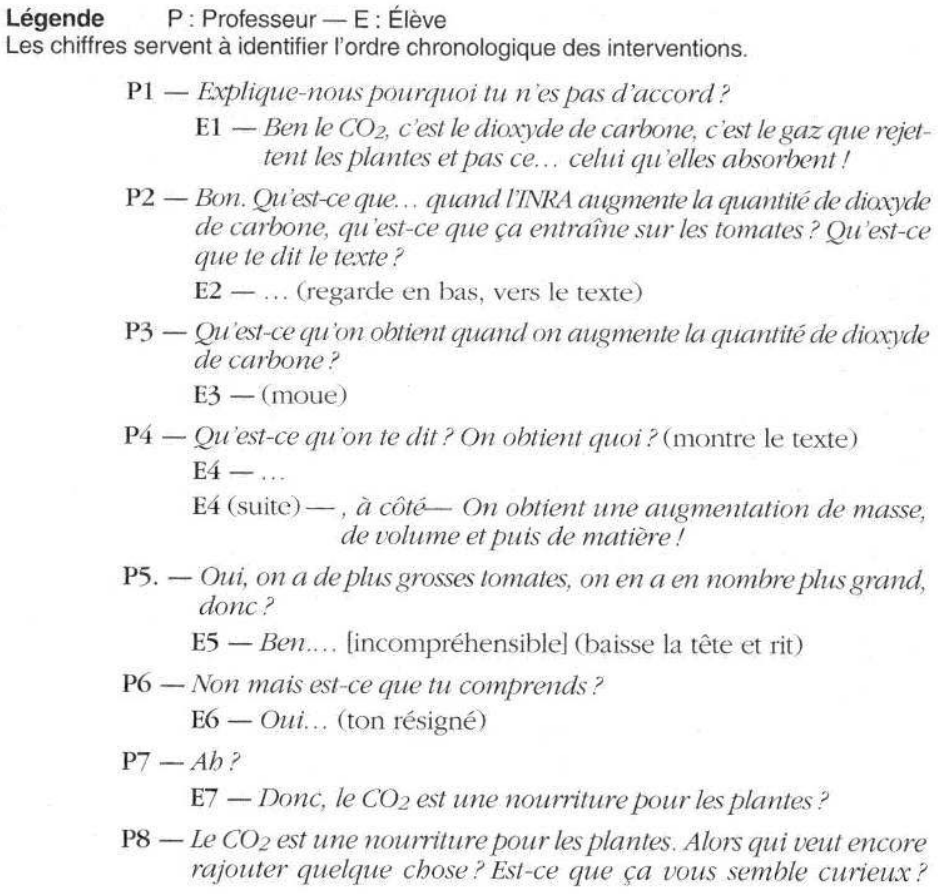

Ce processus de fuite de l'information dérangeante par Pierre-Yves manifeste son désir de cohérence. Gardant le fil de son idée préalable, il nie les faits rapportés tout en en ayant perçu la contradiction potentielle. Face au refus de l'élève d'admettre l'information, et donc à la manifestation de l'idée-obstacle (E1), l'enseignante est décontenancée. Peut-être comme préalable nécessaire dans son esprit à la prise de conscience de la contradiction entre l'idée primitive et l'information nouvelle, elle a recours à l'autorité du document pour la faire accepter (P2). La suite de l'échange vise à faire dire à l'élève lui-même l'information indiscutable puisque donnée dans un document scientifique. Ce que l'élève fait d'un ton interrogateur, manifestant encore son doute (E7). Le doute n'est toujours pas relevé par l'enseignante. Cependant, après avoir confirmé l'information de façon affirmative, l'enseignante renvoie à tout le groupe la question de la contradiction entre cette information et les idées exprimées dans la phase précédente (P8). Or, pour PierreYves, le premier refus de l'information était déjà une réponse : il ne lui est plus possible dans cette nouvelle phase de manifester son doute, disqualifié. Alors que sur l'invitation de l'enseignante, les autres élèves explorent la compatibilité entre l'information nouvelle et leurs idées préalables, il se tait.

L'enseignante intervient pendant un moment en contradiction avec la logique de la situation qu'elle a elle-même mise en place. En effet, l'information relatant les résultats obtenus par l'INRA avait pour fonction de départager les prévisions discutées auparavant par les élèves et de provoquer un conflit cognitif chez ceux pour lesquels ce résultat était contraire aux prévisions. Les interventions de l'enseignante, pendant ce bref moment, empêchent l'explicitation du conflit chez le seul élève pour lequel il se manifeste spontanément. Elle ne tardera pourtant pas à rétablir la logique prévue et à relancer l'explicitation du conflit (P8). Elle semble elle-même prise dans un système de tension entre deux logiques, qui l'incite à balancer entre deux attitudes. L'une peut être 
interprétée en référence à une logique d'appropriation rapide des connaissances, dominante dans l'enseignement, et l'autre au cadre de la recherche qui privilégie le travail sur ce qui s'oppose à cette acquisition. Mais la tension peut aussi être produite par une injonction paradoxale de la recherche : «écoute les idées des élèves, mais fais-les parvenir là où tu veux qu'ils arrivent». L'alternance du professeur entre ces deux logiques traduit peut-être en même temps la nécessité d'un compromis entre la prise en compte du cheminement d'un élève et la conduite du groupe dans son ensemble. Des micro-analyses de ce genre contribuent à modérer les jugements rapides qu'on pourrait faire sur des situations, comme dérives par rapport au projet. On voit ici que la tension du professeur est liée à des aspects multiples dont certains sont incontournables.

\section{Phénomènes inattendus dans l'apprentissage et tensions chez les enseignants}

Des processus normaux dans l'apprentissage, mais qui restent habituellement dans le domaine privé des élèves, sont rendus visibles par le travail sur les obstacles et surprennent les enseignants, qui peuvent avoir des difficultés à réagir de façon à ne pas aller contre la dynamique intellectuelle chez les élèves. Ainsi différentes stratégies de fuite du conflit cognitif ont pu être identifiées, ainsi que des manifestations de régression dans l'apprentissage. Nous nous attarderons plus particulièrement sur ces dernières. Ce sont des processus auxquels on peut s'attendre, ils sont décrits par des psychologues (Piaget) comme par des didacticiens (Arsac et al., 1988). Nous avions d'ailleurs prévu dans notre schéma théorique de l'évolution des obstacles chez les élèves (Astolfi, Peterfalvi, 1993) deux moments de régression: le premier étant le signe d'une déstabilisation provoquée par le conflit et la perte des repères que constituaient les idées-obstacles; le deuxième correspondant à un passage à la parole personnelle où l'apprenant s'approprie comme il peut en les réorganisant à sa façon les nouvelles idées (intériorisation). Ces régressions sont indicatrices d'un changement conceptuel en cours. On rencontre également un autre type de régressions, qui correspondent en réalité à un changement dans les questions scientifiques posées, mais sont interprétées comme des régressions quand ce changement n'est pas vu.

Cependant, pour les enseignants, cela constitue souvent une surprise de constater que les élèves, à certains moments, produisent des explications en retrait par rapport à ce qui semblait avoir été construit, et que les obstacles qui semblaient dépassés resurgissent. Et ce constat s'accompagne d'une inquiétude et d'un sentiment d'échec. Il faut tout le recul que peut proposer un groupe de recherche pour parvenir à relativiser l'échec et à admettre que les régressions observées sont nécessaires et/ou inévitables. Le problème est que, dans le feu de l'action, l'enseignant qui constate une régression et la vit comme un échec de son action antérieure, peut être tenté de court-circuiter la pensée des enfants en imposant la solution conceptuelle qui lui paraît évidente et qu'il pensait déjà acquise par les élèves, comme s'il suffisait de rafraîchir leur mémoire, comme si c'était un défaut d'attention ou un oubli.

16 C'est ce qui se passe dans cette classe de troisième qui, à la suite de manipulations variées, avait utilisé deux modèles de la matière élaborés précédemment pour représenter les types de transformations, changements d'état et réaction chimique. Ces modèles figuraient sous forme d'affiches dans la classe et pouvaient servir de référence dans le 
cours du travail. Lors d'une étape ultérieure du travail où il était demandé aux élèves de préciser l'origine des bulles dans trois phénomènes différents (eau gazeuse qu'on agite, eau qu'on fait bouillir et électrolyse de l'eau) et d'identifier les types de transformation, un groupe d'élèves discutant avec l'enseignante ne sait plus mobiliser la notion de changement d'état. Après coup, en analysant l'enregistrement vidéo, nous avons pu avancer une interprétation de cette réaction en régression sur les performances antérieures. Elle révèle probablement que la notion de changement d'état s'était trouvée déstabilisée par la construction de la notion de réaction chimique et n'avait pas été reconstruite de façon satisfaisante, ce qui était passé inaperçu sur le moment.

Mais sur le moment, l'enseignante, qui ne dispose pas de cette grille de lecture, se trouve désarçonnée par les hésitations et erreurs des élèves, et tente de leur faire redire ce qu'ils avaient dit, et croyait-elle, bien acquis dans une séance précédente. Les élèves, eux, sentent que le professeur est déçu mais ne savent pas ce qu'elle attend d'eux puisqu'ils ont changé de système de pensée et n'identifient plus aussi nettement la situation « changement d'état ».

Un autre exemple nous vient d'une classe de seconde dans un contexte de travail voisin de celui qui a été présenté plus haut sur la croissance des tomates. Après avoir prévu par écrit l'effet des différents taux de dioxyde de carbone que l'INRA avait fait varier dans une expérience de culturede tomates, les élèves en débattent et argumentent leurs avis. Puis individuellement ils écrivent leurs nouvelles prévisions. Or, l'analyse ultérieure de leurs écrits le montre, trois élèves dont les premières prévisions étaient correctes se rangent à l'avis d'une de leurs camarades lors de la discussion de groupe et font maintenant des prévisions incorrectes. La discussion des prévisions, qui avait été mise en place dans l'idée de mettre en contradiction les idées non conformes, aboutissait au contraire à disqualifier les idées correctes. 
Réflexions et discussion d'élèves de seconde mentionnées ci-dessus.

\author{
Légende En caractères italiques: Les élèves \\ En caractères romains: Commentaires de l'auteur
}

Écrit 1 de Juliette : le schéma de plan de tomate, sur lequel on demandait d'indiquer comment la plante se nourrit, ne fait pas figurer d'échanges gazeux. L'idée de l'intervention de gaz n'est pas immédiatement disponible dans le contexte de la nutrition.

Écrit 2: "Il faut une valeur supérieure à 0,003\% pour que l'échange s'opère plus vite et en plus grande quantité, ainsi la plante poussera plus vite ". Lorsque des gaz sont évoqués, comme ici dans le dispositif expérimental, la prévision du résultat est correcte, mais sans que le sens de l'échange soit spécifié. C'est probablement une réponse "récitée " et non appropriée.

Écrit 3: "Je pense qu'Audrey a raison. On doit avoir une valeur inférieure à $0,003 \%$ parce que sinon, il y aurait trop de $\mathrm{CO}_{2}$ et pas assez de $\mathrm{O}_{2}$, et la plante s'asphyxierait comme l'être humain ". Dans la discussion, les arguments d'une autre élève ont réactivé un obstacle latent relatif au rôle respiratoire des gaz, l'analogie avec l'homme jouant comme argument supplémentaire. Juliette abandonne la réponse apprise et récupère sa propre pensée.

Cette élève réussira très bien par la suite à intégrer le rôle du dioxyde de carbone dans la nutrition et dans la respiration de la tomate.

L'enseignante, si elle s'en était aperçue immédiatement, aurait pu se trouver déstabilisée et tenter de réhabiliter d'une façon ou d'une autre la première réponse. Mais la situation était telle qu'elle n'en a pris connaissance qu'après coup, et la réaction d'inquiétude que cette réponse aurait pu provoquer n'a pas eu lieu. Par la suite, on voit que ces trois élèves seront capables de mettre en contradiction leur propre idée et d'avancer par rapport à l'idée-obstacle. L'analyse conduite dans le cadre collectif du groupe de recherche, comprenant l'enseignante concernée, nous a conduit à interpréter ce recul comme une régression nécessaire au travail de l'obstacle: l'expression de leur système de pensée personnel a donné aux élèves la possibilité de le modifier par la suite.

D'une façon générale, ces phénomènes qui surprennent les enseignants au départ constituent un point de cristallisation des tensions à l'œuvre dans la conduite de ces séquences pour les enseignants entre des aspects de modèles classiques d'apprentissage et d'enseignement, auxquels ils continuent d'adhérer en parallèle ou qui conditionnent le contexte général de l'enseignement et dont il faut bien qu'Os tiennent compte, et les aspects du modèle constructiviste sur lesquels la recherche s'appuie. Les réactions de déception et d'inquiétude peuvent être comprises par l'attente d'une réussite linéaire et immédiate, en accord avec la conception de l'apprentissage comme un processus cumulatif linéaire. Ou encore par la mise en évidence des acquisitions réellement disponibles, qui restent habituellement masquées sous le niveau moyen de la classe. Ou par l'attente d'un transfert spontané d'une acquisition dans un contexte donné à un autre contexte, avec changement de niveau de généralité souvent, ou encore introduction d'une plus grande complexité et donc mobilisation d'un réseau conceptuel plus étendu. La déception est d'autant plus vive qu'on a investi beaucoup de temps et d'énergie pour mettre en place une séquence bien construite et visant un progrès intellectuel significatif. 
Que faire alors? Comment éviter les écueils symétriques : court-circuiter les régressions apparentes et peut-être passer à côté de l'obstacle et d'une appropriation de la construction conceptuelle; s'y enliser et le résultat est le même? Une meilleure interprétation des régressions observées, comme celles que nous avons développées dans la recherche, permet aux enseignants de mieux évaluer en situation le déplacement des problèmes ou la réorganisation en cours qu'elles indiquent, et de trouver des réponses adaptées. Ils en témoignent largement au cours d'entretiens conduits après la fin de la recherche. À cela peuvent s'ajouter des réactions de satisfaction, car c'est signe que les obstacles choisis sont de vrais obstacles qui résistent et que le travail entrepris est donc déterminant pour l'apprentissage.

\section{Quel statut donner aux décalages et imprévus ?}

La plupart des analyses de cette recherche sont produites dans des groupes d'enseignants qui ont pour tâche une discussion critique de leurs essais ou dans des groupes associant les chercheurs et les enseignants. Cette organisation permet une double réélaboration: celle du projet théorique propositionnel, à laquelle les enseignants sont associés et celle des situations de travail que les enseignants remanient au cours d'essais successifs.

Le schéma suivant peut rendre compte de la dialectique des relations entre théorisation et réalisations concrètes.

Figure 2 : La dialectique du cadre théorique et des réalisations

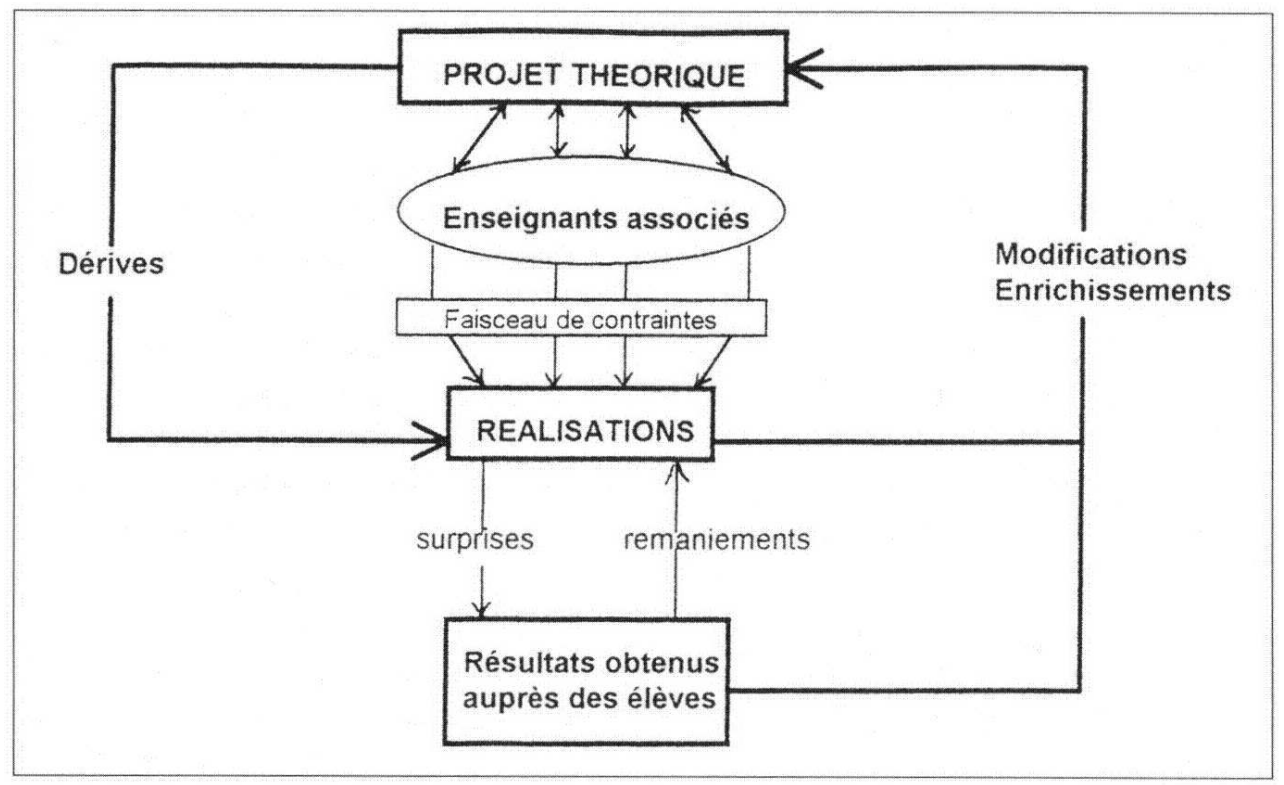

\subsection{Une prise en compte nécessaire}

Partons de la façon dont sont pris en compte les résultats obtenus auprès des élèves. Ils le sont de différentes manières : par les enseignants pour reconsidérer leur séquence et la reconstruire avec l'aide que peut apporter le cadre théorique, ou pour réajuster au fur et à mesure leur action, sur un mode régulatoire; mais aussi plus globalement par la 
recherche, pour moduler le cadre propositionnel et fournir des connaissances beaucoup plus précises sur les processus intellectuels en jeu.

Certains écarts entre ces résultats et les attentes des enseignants, qu'ils considèrent parfois comme signes d'échec de leur stratégie, peuvent être réinterprétés à la lumière du cadre théorique comme des phases positives, indispensables à l'apprentissage et à la remise en cause des systèmes d'idées constitués en obstacles. Nous l'avons vu à propos des "régressions apparentes». Dans ce cas, le cadre théorique intervient dans la compréhension des processus intellectuels et permet de produire des analyses qui constitueront des outils pour l'enseignement.

Dans d'autres cas, c'est le projet théorique qu'il convient de modifier et d'enrichir. C'est ainsi qu'en raison de la persistance de manifestations des obstacles après la mise en œuvre de situations favorisant principalement les conflits sociocognitifs, nous avons été conduits à donner une place croissante à des activités visant une prise de conscience des obstacles par les élèves a posteriori, une fois qu'ils semblent les avoir dépassés dans un contexte local. Sur le plan propositionnel ceci relativise la place des conflits sociocognitifs comme moteurs dans le processus d'abandon des idées-obstacles. Pour cette même raison, nous avons modulé notre vocabulaire: plutôt que de "franchissement" des obstacles, nous parlons plus volontiers de " travail sur les obstacles». Dans ce sens, nous en savons davantage après réalisation sur les stratégies de résistance des obstacles et donc sur les modalités possibles de contre-stratégies.

Bien sûr, les résultats obtenus auprès des élèves ne peuvent pas être pris en compte de la même manière lorsque les séquences s'éloignent trop du projet. Lorsqu'on est en face d'une dérive telle que le travail sur les obstacles n'est plus perceptible, on ne peut rien en conclure quant à la pertinence du cadre théorique. Ces résultats peuvent en revanche être utilisés par les enseignants pour remanier leur séquence dans un sens qui les rapproche davantage du projet. Par exemple, lorsque des évolutions décevantes d'élèves attirent l'attention sur le fait que le problème posé n'en était pas vraiment un pour eux et que par conséquent ils ne se sont engagés dans aucun véritable travail intellectuel, des réajustements dans ce sens peuvent être opérés.

Les productions des élèves interviennent aussi pour renseigner les enseignants sur la pertinence de leurs choix par rapport aux particularités de leurs classes. L'objectifobstacle choisi peut ne pas être pertinent pour une population donnée. Les productions et propositions des élèves sont source de réajustements en situation. Mais bien plus, leur prise en compte en cours de situation de travail s'avère primordiale pour l'enrôlement des élèves. Le travail sur les obstacles, qui par sa nature même suppose un engagement important de leur part, exige qu'ils sentent leurs idées sérieusement prises en compte. C'est la raison pour laquelle la flexibilité de ces situations est essentielle pour leur réussite.

\subsection{Avantages théoriques et risques}

Si on considère maintenant ce que peut apporter au cadre théorique tant la structure des séquences proposées par les enseignants que leur façon de les mener,notre attention est plutôt attirée sur le caractère réalisable du projet au regard des contraintes scolaires et des conceptions des enseignants sur l'apprentissage et l'enseignement. 

théorique et par là même le précisent. C'est ainsi qu'on est amené à se questionner sur le caractère dissociable ou non, dans une chronologie, des phases de " déconstruction » et de "construction "; ou encore à se questionner sur les différentes façons de combiner une séquence courte, répondant aux exigences du projet, avec des reprises à plus long terme. Elles permettent de comprendre comment certaines modalités de travail induisent certains parcours intellectuels : par exemple, conforter dans un premier temps l'obstacle à travailler en mettant en lumière ce en quoi le système d'idées qu'il constitue est pertinent (faire par exemple des expériences pour montrer que les végétaux se nourrissent bien de sels minéraux et d'eau, ou bien faire discuter ensemble ceux qui sont dans le même système d'idées) permet un engagement ultérieur plus important des élèves. projet, sur les contradictions éventuelles dans lesquelles celui-ci peut les engager, sur les aspects qui leur sont plus difficilement accessibles ou acceptables. Elles montrent par exemple que l'importance des conditions de mise en scène sociale pour engager les élèves dans une dynamique d'évolution de la pensée personnelle a tendance à ne pas leur apparaitre de façon immédiate. Elles manifestent aussi la difficulté pour les enseignants de s'approprier l'idée même d'obstacle, qui peut leur sembler trop négative et qu'ils ont tendance dans un premier temps à confondre avec celle d'idée fausse ou de lacune. déroule éclaire sur les compétences qui leur sont nécessaires et sur la direction des efforts de formation à fournir. previsible puisque les cadres de leur pensée y sont remis en question, mais aussi pour les enseignants : la recherche nous a davantage informés sur ce point. On peut voir en effet un parallélisme dans ce qui se passe pour les élèves et les enseignants, qui reprennent à leur propre compte le terme de " déstabilisation » qu'ils employaient au départ à propos des élèves. Ce travail contribue en effet à un processus important de formation pour eux. Ils sont conduits à remettre en question de façon parfois essentielle leur manière habituelle d'enseigner, ce qui peut engendrer un sentiment d'insécurité.

D'une manière plus générale, le discours que les enseignants tiennent à propos de la mise en œuvre des situations de travail met l'accent sur le fait qu'elles leur sont coûteuses, tant du point de vue de la préparation que de la disponibilité qu'elles exigent en cours de réalisation, du fait de l'adaptation permanente qu'elles demandent aux idées des élèves. En effet, la flexibilité requise par ce type de travail exige une attention constante et des compétences particulières de leur part, dont ils ne disposent pas nécessairement au départ, mais que la participation à la recherche contribue à leur faire acquérir. C'est une des limites de ce travail, mais sur laquelle les résultats de la recherche peuvent avoir un impact : ils contribuent à préciser le "bagage » nécessaire des enseignants pour ce genre de situations et à le leur fournir. Ainsi, un répertoire de tâches et de modes d'intervention possibles est peu à peu constitué, les obstacles interférant avec le champ conceptuel étudié sont progressivement précisés, les processus cognitifs qui interviennent dans la résistance des obstacles sont mis en lumière. 


\section{Conclusion}

35

À l'issue de cette recherche, on se trouve face à une série d'interrogations ou d'alternatives, susceptibles d'orienter de nouvelles pistes de recherches.

Face aux difficultés rencontrées quant au dépassement des obstacles par les élèves, plusieurs solutions sont possibles.

On peut raffiner les dispositifs, chercher des situations susceptibles d'être plus efficientes. Dans cette perspective, on pourrait explorer de façon plus intensive les activités visant à développer chez les élèves une capacité à reconnaître certains obstacles en même temps qu'une attitude de vigilance critique vis-à-vis de leur propre pensée. On peut aussi chercher de meilleures articulations entre activités centrées sur les obstacles et autres activités didactiques. Quoi qu'il en soit, les situations explorées ont montré qu'il est important de limiter l'illusion de maîtrise des processus dans ce type d'activités. Il serait vain de trop attendre d'une situation unique et l'idée de franchissement d'obstacles à moyen ou long terme semble plus pertinente. Par ailleurs, est-il possible de s'engager dans une telle direction sans diminuer la faisabilité didactique des dispositifs?

On peut choisir de tourner plutôt l'attention sur les problèmes rencontrés par les enseignants et explorer davantage les pistes susceptibles de les résoudre. Une des solutions consisterait alors à chercher des situations moins coûteuses pour les enseignants et à leur fournir des outils susceptibles de leur faciliter la tâche. Mais nous sommes là face à une nouvelle interrogation. Dans quelle mesure peut-on fournir des outils réellement utilisables par les enseignants, sans qu'ils aient eux-mêmes participé à leur élaboration? Peut-on faire l'économie du processus de formation dont la recherche a été l'occasion pour ceux qui y ont été associés? Par ailleurs ne risque-t-on pas de diminuer l'investissement des enseignants et d'affaiblir en ricochet l'impact des situations proposées sur l'évolution des élèves? Le problème est de trouver un coût didactique optimum: suffisant pour initier une dynamique de changement, sans être excessif pour n'être pas dissuasif.

Avec l'exploration des limites et l'interrogation sur les efforts à fournir pour rendre le projet réalisable, le problème plus général que nous voulons soulever est celui de la position que la recherche en didactique peut adopter dans le système de tension entre utopie et réalité.

BIBLIOGRAPHIE

\section{Références citées}

ARSAC G., GERMAIN G. \& MANTE M., Problème ouvert et situation-problème, Villeurbanne, IREM de Lyon, 1988. 
ASTOLFI J.-P., « Trois paradigmes pour les recherches en didactique », in Revue française de pédagogie, $\mathrm{N}^{\circ} 103,1993$.

ASTOLFI J.-P. \& PETERFALVI B., « Obstacles et construction de situations didactiques en sciences expérimentales », in Aster, $\mathrm{N}^{\circ} 16,1993$.

MARTINAND J.-L., " La référence et le possible dans les activités scolaires », in Actes de l'Atelier international de Didactique de la Physique (La Londe des Maures 1983), 1984.

\section{Références complémentaires}

ASTER (équipe), Procédures d'apprentissage en sciences expérimentales, Paris, INR, 1985.

ARCÀ M. \& CARAVITA S., " Le constructivisme ne résoud pas tous les problèmes ", in Aster, $\mathrm{N}^{\circ}$ $16,1993$.

BACHELARD G., La formation de l'esprit scientifique, Paris. Vrin, 1938.

BEDNARZ N. \& GARNIER C.(eds), Construction des savoirs, obstacles et conflits, Ottawa, 1989.

C1RADE, Agence d'Arc.

FABRE M., Bachelard éducateur, Paris, PUF, 1995.

FAVRE D., « Conception de l'erreur et rupture épistémologique », in Revue Française de Pédagogie, $\mathrm{N}^{\circ} 111,1995$.

LAROCHELLE M. \& DESAUTELS J., Autour de l'idée de science, Québec, Ottawa, Les Presses de l'Université Laval, 1992.

MEIRIEU P., La pédagogie entre le dire et le faire, Paris, ESF, 1995.

NUSSBAUM J. \& NOVICK S., “Alternative frameworks, conceptual conflict and accommodation : toward a principled teaching strategy", in Instructional Science, $\mathrm{N}^{\circ} 11,1982$.

ORANGE C., « Le concept d'obstacle en didactique des sciences : nécessité d'une approche plurielle ", in Le problème et l'obstacle en didactique des sciences, Documents du CERSE, $\mathrm{N}^{\circ} 60$, Caen, Université de Caen, 1993.

PETERFALVI B. \& VÉRIN A., "Tackling conceptual obstacles to acquiring scientific knowledge in a constructivist teaching model", in Actes du séminaire AEDB - septembre 1994, Chamonix-Genève : in A. Giordan \& Y. Girault, The new learning models, Nice, France, Z' edition, 1996, p.125-134.

PETERFALVI B., « Activités réflexives d'élèves en classe de sciences : des compétences méthodologiques au travail sur les obstacles ", In Giordan A., Martinand J.-L. \& Raichvarg D., Que savons-nous des savoirs scientifiques et techniques ?, Actes JIES, XVII, 1995.

PLÉ E., «Objectif-obstacle et gestion du conflit sociocognitif : difficultés liées à la reprise d'un dispositif didactique flexible ", in Actes du $5^{\mathrm{e}}$ Séminaire National de la Recherche en Didactique des Sciences Physiques, octobre 1995, Reims, 1995.

VÉRIN A. \& PETERFALVI B., « Fonctions de l'écriture dans le travail d'obstacles en classe de sciences », In Giordan A., Martinand J.-L. \& Raichvarg D., L'alphabétisation scientifique, Actes JIES XVI, 1994.

VÉRIN A., « Mettre par écrit ses idées en sciences pour les faire évoluer », in Repères, (sous presse). 


\section{RÉSUMÉS}

Plutôt qu'un exposé sur des résultats de recherche, nous aimerions proposer ici une réflexion épistémologique que la pratique d'une activité de recherche nous a conduites à mener. Notre recherche pose le problème des dissonances entre les constructions théoriques de départ et les mises en oeuvre réelles en classe. Comment interpréter ces dissonances, quel statut leur donner, quelles conclusions en tirer par rapport à la recherche ? C'est autour de cet ensemble de problèmes que nous présentons ici quelques éléments de réflexion.

Rather than an expose of research results, we would here like to propose an epistemological reflection which the exercice of research activity bas lead us to, follow. Our research lays out the problem of the discrepancy between original theoretical constructions and their concrete day to day application in the classroom. How is one to interpret these discrepancies? How are they to be classed? What conclusions can one draw from these discrepancies as concerns the underlying research? It is around this set of problems that we here present a few elements of reflection.

\section{INDEX}

Mots-clés : didactique, épistémologie, obstacle, traitement didactique

Keywords : didactic, epistemology, implementation of a didactic theory

\section{AUTEURS}

\section{BRIGITTE PETERFALVI}

Institut National de Recherche Pédagogique, Paris

\section{ANNE VÉRIN}

Institut National de Recherche Pédagogique, Paris 